\title{
Médiévales
}

Langues, Textes, Histoire

50 | printemps 2006

Sociétés nordiques en politique (XII ${ }^{\mathrm{e}}-\mathrm{XV}^{\mathrm{e}}$ siècles)

\section{La fascination et l'action à distance : questions médiévales (1230-1370)}

Fascination and action at a distance : medieval questions (1230-1370).

\section{Béatrice Delaurenti}

\section{(2) OpenEdition}

12 Journals

\section{Édition électronique}

URL : https://journals.openedition.org/medievales/1420

DOI : $10.4000 /$ medievales. 1420

ISSN : 1777-5892

Éditeur

Presses universitaires de Vincennes

\section{Édition imprimée}

Date de publication : 1 juin 2006

Pagination : 137-154

ISBN : 2-84292-186-0

ISSN : 0751-2708

\section{Référence électronique}

Béatrice Delaurenti, « La fascination et l'action à distance : questions médiévales (1230-1370) », Médiévales [En ligne], 50 I printemps 2006, mis en ligne le 15 septembre 2008, consulté le 22 avril 2022. URL : http://journals.openedition.org/medievales/1420 ; DOI : https://doi.org/10.4000/ medievales. 1420

Ce document a été généré automatiquement le 22 avril 2022.

Tous droits réservés 


\title{
La fascination et l'action à distance : questions médiévales (1230-1370)
}

Fascination and action at a distance : medieval questions (1230-1370).

\author{
Béatrice Delaurenti
}

1 Que le serpent tue sa proie en la regardant fixement, comment concevoir un tel prodige? Qu'une femme voie apparaître dans le miroir un nuage de sang quand elle a ses règles, comment expliquer pareil phénomène ? Y a-t-il une cause naturelle qui puisse rendre compte de ce mode d'action à distance? Ces questions ont fait débat dans les universités médiévales.

2 Le terme fascinatio désigne un pouvoir d'influence propre à certains animaux ou certains êtres humains, un pouvoir qui réside dans la seule puissance du regard, sans autre forme de contact. L'idée que la fascination aurait une cause naturelle est issue d'une nouvelle conception de la nature, alimentée par des penseurs du milieu chartrain au XII ${ }^{\mathrm{e}}$ siècle, étayée ensuite par la redécouverte de la philosophie naturelle d'Aristote au XIII ${ }^{e}$ siècle. Avant le XII ${ }^{\mathrm{e}}$ siècle, la natura était considérée comme opus creatoris et traitée comme un réservoir de symboles; rien ne justifiait une recherche de causes naturelles ${ }^{1}$. À partir des $\mathrm{XII}^{\mathrm{e}}$-XIII ${ }^{\mathrm{e}}$ siècles, une nouvelle définition de l'action naturelle paraît possible. La Physique d'Aristote ${ }^{2}$ énonce en effet qu'une action est naturelle si elle met en contact un moteur et un mobile : tout mouvement naturel procèderait par contact.

3 La Physique fut traduite à partir du grec entre 1130 et 1150, puis à partir de l'arabe dans la deuxième moitié du XII ${ }^{\mathrm{e}}$ siècle ${ }^{3}$. Mais l'enseignement de la Physique avait été interdit à l'Université de Paris en 1210 et en 1215, et l'ouvrage ne fut officiellement inscrit au programme de la faculté des arts qu'en 1255. Sa diffusion fut donc tardive. Dans les années 1260-1270, les traductions révisées de Guillaume de Moerbeke fournirent une nouvelle version latine des textes aristotéliciens, plus claire et plus exacte. La seconde moitié du XIII siècle aura été marquée par ce "nouvel Aristote", désormais connu en profondeur et en détail par les maîtres des facultés des arts et de théologie. Au 
XIV siècle, la référence à la Physique est devenue explicite pour définir l'action naturelle.

4 La notion d'action à distance se heurtait à la conception scolastique de l'action naturelle, fondée sur la théorie du contact formulée par Aristote ${ }^{4}$. Les débats doctrinaux de l'époque médiévale sur la fascination constituent ainsi un excellent observatoire pour comprendre de quelle façon les auteurs envisageaient l'action naturelle et ses limites.

Nous analyserons ici les réflexions de quatre auteurs appartenant à l'époque scolastique. De l'essor intellectuel des $\mathrm{XII}^{\mathrm{e}}$-XIII ${ }^{\mathrm{e}}$ siècles jusqu'à la fin $\mathrm{du}_{\mathrm{Xv}}$ siècle, la période fut marquée par une exigence de rigueur logique et le souci d'articuler la raison humaine à la foi chrétienne. C'est dans ce cadre que la question de la fascination a été posée par Guillaume d'Auvergne, Roger Bacon, Thomas d'Aquin et Nicole Oresme. Chacun d'eux, maîtres séculiers ou membres des ordres mendiants, a enseigné pendant un temps à l'université de Paris. Leurs écrits ont marqué l'histoire intellectuelle de l'Occident médiéval et leur influence fut décisive, notamment en philosophie naturelle. Vers 1230, dans le De universo, Guillaume d'Auvergne inscrit la question de la fascination dans son analyse des propriétés occultes des animaux et des choses naturelles ${ }^{5}$. Dans les années 1266-1268, Roger Bacon étudie la fascination dans son Opus maius $^{6}$. À la même époque, Thomas d'Aquin aborde la question par le biais de la doctrine d'Avicenne sur le pouvoir de l'âme dans le Contra Gentiles et dans la Summa theologica ${ }^{7}$. Enfin, dans les années 1351-1362, Nicole Oresme fait le point sur la fascination dans le De configuratione qualitatum ${ }^{8}$; il reprend la question en 1370 dans le De causis mirabilium ${ }^{9}$.

6 Ces quatre auteurs ont en commun de saisir l'action à distance dans une perspective résolument philosophique, avec la volonté de trouver une explication naturelle à ce phénomène. Ils participent, chacun à leur manière, d'une même tentative d'expliquer le monde par la voie de la raison. Sur la fascination, ils font appel aux mêmes autorités et aux mêmes exemples; les positions de Roger Bacon ou de Nicole Oresme témoignent de l'influence des idées de Guillaume d'Auvergne en matière de philosophie naturelle. En outre, nos quatre auteurs ont la particularité de porter leur réflexion à la fois sur le pouvoir du regard et sur celui de la voix : dans les débats de leur époque sur la virtus verborum, ils ont tenté de savoir si le pouvoir des incantations relevait de la nature ${ }^{10}$. Pour eux, le pouvoir du regard et le pouvoir de la voix posent une seule et même question : où commence, où s'arrête l'action naturelle?

7 À la fin du Moyen Âge, la question de la fascination entre dans le champ médical. Dès 1310, le médecin Pietro d'Abano lui fait une place dans le Conciliator, une vaste encyclopédie dans laquelle il entreprend de réconcilier médecine et philosophie ${ }^{11}$. Puis l'intérêt des médecins va croissant pour le regard comme instrument du pouvoir de l'âme sur le corps; le mauvais œil est considéré comme un problème médical ${ }^{12}$. Notre étude du versant philosophique et théologique des débats sur la fascination se situe en amont de cette littérature médicale.

Définition et exemples de fascination

Dans les textes scolastiques, le terme fascinatio est régulièrement illustré par les mêmes citations et les mêmes anecdotes. Elles permettent de se faire une idée du phénomène tel que le concevaient les auteurs qui en étudiaient les causes. Deux passages de la Bible sont récurrents : le Livre de la Sagesse, 4, 12 et l'Épitre de Saint Paul aux Galates, 3, 1. Dans ces textes, le terme fascinatio prend un sens général, il désigne l'influence maléfique 
qu'exerce une âme mauvaise; le rôle intermédiaire du regard n'est pas précisé. Le verset de l'Épitre aux Galates est accompagné, dans la Glose ordinaire, d'un commentaire sur le sens de fascinatio: le mot désignerait précisément une action qui passe par les yeux, qui serait liée à un trouble de l'âme et qui aurait pour cible les personnes les plus fragiles ${ }^{13}$. C'est ainsi que les théologiens et philosophes des $\mathrm{XIII}^{\mathrm{e}}$ et $\mathrm{XIV}^{\mathrm{e}}$ siècles comprenaient la fascination: une influence qui fait appel au seul pouvoir du regard. Il n'y a pas de contact, c'est une action à distance.

Outre les citations bibliques, quatre exemples donnent une image précise de la fascination: le basilic, la femme au miroir, les agneaux de Virgile et la chute du chameau.

\section{Le serpent et la femme}

Le basilic est un serpent qui tue. Ce n'est pas seulement la morsure du serpent qui est mortelle, mais aussi son souffle, son odeur et surtout son regard : l'homme qui croise ce regard en meurt. Pline mentionne l'animal à plusieurs reprises dans les Histoires. Il le décrit comme un serpent qui sème la mort autour de lui par son sifflement et son haleine ${ }^{14}$; il complète cette description en soulignant le pouvoir de son regard ${ }^{15}$. On retrouve l'exemple du basilic dans les textes de l'Antiquité tardive : chez Lucain ${ }^{16}$ et chez Solin ${ }^{17}$, lequel ne fait aucune mention du regard du serpent. Au Moyen Âge, le renom de l'animal est véhiculé par les encyclopédistes. À la fin du XII siècle, Alexandre Neckham évoque le basilic dans le De naturis rerum, mais il ne dit rien de la puissance de son regard ${ }^{18}$. Vers 1240 , Thomas de Cantimpré lui consacre un paragraphe du De natura rerum et insiste, en s'appuyant sur Pline, sur le regard foudroyant de l'animal ${ }^{19}$. Vers 1250 , Vincent de Beauvais ne lui consacre pas moins de trois chapitres dans le Speculum naturale. Il reprend l'ensemble des références antiques et médiévales sur le basilic, mentionne le sifflement du serpent et surtout son regard, fatal pour l'homme même quand il se trouve à bonne distance ${ }^{20}$.

Le regard fascinant du basilic connaît une fortune particulière, au XIII ${ }^{e}$ et au XIV siècle, dans les réflexions doctrinales sur la magie et les prodiges de la nature. La fréquence de cette citation dans les textes médiévaux en fait un paradigme de la fascination. Guillaume d'Auvergne utilise l'exemple du basilic à la manière d'un étalon qui permet de mesurer les forces occultes de la nature ${ }^{21}$. Dans l'Opus maius, Roger Bacon présente le serpent basilic comme une arme de guerre. Une ville assiégée par Alexandre avait placé un serpent basilic sur les murailles afin de foudroyer les assaillants. Sur le conseil d'Aristote, Alexandre disposa de grands miroirs pour renvoyer le regard empoisonné du serpent, " de sorte que celui-ci fut détruit par son propre venin ${ }^{22}$ ». Bacon se réfère à la lettre qu'Alexandre aurait envoyée à Aristote pour lui rapporter les épisodes de la campagne d'Inde; dans ce récit, il est possible qu'il se soit inspiré de l'épisode dit de «la nuit des terreurs ${ }^{23}$ :

À la troisième heure de la nuit, des serpents à deux ou trois têtes ornées d'une crête, de la taille d'une colonne, parfois plus grands, arrivèrent des grottes des montagnes voisines; ils avaient des langues fourchues, un regard étincelant et venimeux, et leur souffle aussi était empoisonné ${ }^{24}$.

Le pouvoir de ces serpents fantastiques est exactement celui du basilic, leur regard mortel aurait semé la terreur dans l'armée d'Alexandre.

Du serpent à la femme, il n'y a qu'un pas dans les textes doctrinaux. Le thème du mauvais œil féminin se trouve déjà chez Guillaume d'Auvergne. Le pouvoir de fascination par le regard n'est pas rapporté à un animal mais à un homme ou une 
femme, avec une préférence pour les vieilles femmes, les vetule, qui sont accusées par ailleurs de pratiquer des maléfices ${ }^{25}$. Guillaume d'Auvergne se fonde implicitement sur quelques vers des Amours d'Ovide: les attributs de la vetula sont les mauvaises incantations et surtout le regard, car elle possède une double pupille ${ }^{26}$. La femme à la double pupille d'Ovide est un topos de la fascination. On la trouve aussi dans l'Epistola de secretis operibus attribuée à Roger Bacon ${ }^{27}$; au XIv siècle, Oresme l'évoque encore dans le De configuratione ${ }^{28}$.

Le thème du mauvais œil féminin rejoint un autre motif de fascination fondé sur Aristote, celui de la femme au miroir. Il est présent dans les sources doctrinales à partir de la seconde moitié du XIII ${ }^{e}$ siècle, lorsque la philosophie aristotélicienne commence à être intégrée dans la pensée occidentale. La formulation originelle se trouve dans le traité De somnis :

Sur des miroirs tout à fait nets, quand les femmes se regardent au moment des menstrues, il se forme à la surface comme un nuage de sang. Si le miroir est neuf, il n'est pas facile de faire disparaître une telle tache ; s'il est ancien, c'est plus aisé ${ }^{29}$. Selon Aristote, les organes des sens ne se contentent pas de subir certains effets, ils agissent en fonction des plus infimes variations du corps humain. C'est le regard qui est actif, qui agit par contact sur l'espace aérien.

Le prodige de la femme au miroir est signalé par Roger Bacon dans l'Opus maius ${ }^{30}$. Le pouvoir de la femme menstruée est comparable à la contagion de certaines maladies comme la lèpre : comme la femme en période menstruelle, le lépreux est dans un état d'impureté. L'un et l'autre infectent l'air et le contaminent par des émanations létales ${ }^{31}$. Thomas d'Aquin reprend le même exemple quand il analyse la fascination, mais il le réduit à une anecdote minimale : la tache de sang et l'infection de l'air ne sont pas mentionnés ${ }^{32}$. Quant à Nicole Oresme, il juxtapose l'exemple de la femme au miroir et celui du serpent ${ }^{33}$. La scène de la femme au miroir, régulièrement associée aux maléfices du basilic, serait donc un exemple de base, comme si le mauvais œil de la femme menstruée était l'équivalent humain du regard fatal du serpent. Dans les écrits sur la fascination comme dans la tradition scripturaire, la malignité de la femme est parallèle à celle du serpent.

Les agneaux, le chameau et la mule

17 Un autre exemple récurrent de fascination est tiré des Bucoliques de Virgile ${ }^{34}$. Un jeune berger, Ménalque, se plaint de la maigreur de ses moutons, il pense que leur état est un effet de la fascinatio. La phrase de Ménalque, «je ne sais quel œil fascine mes tendres agneaux », est citée par Roger Bacon et par Nicole Oresme ${ }^{35}$.

18 Un dernier exemple revient régulièrement : l'histoire de la chute du chameau. Pour mieux en saisir la portée, il faut se reporter à la formulation qu'en donne, au XI siècle, le théologien arabe Algazel (al-Ghazzālī, 1058-1111) :

Un œil peut envoyer un homme dans un fossé et un chameau dans une étuve, et on dit que ces hommes ont été fascinés ${ }^{36}$.

Guillaume d'Auvergne fait une mention rapide de cette anecdote, mais le médium oculaire n'est pas mentionné : il n'est pas question de fascination, la chute du chameau illustre le pouvoir de l'imagination ${ }^{37}$. En 1277 , l'exemple du chameau est cité parmi les 219 propositions condamnées à Paris par l'évêque Étienne Tempier; l'article 112 nie qu'un magicien (incantator) soit capable de projeter un chameau dans une fosse par son seul regard ${ }^{38}$. Le terme incantator fait supposer que la chute du chameau aurait été le résultat d'une incantation. Mais il semble que le médium importe peu : qu'il s'agisse de 
la voix ou du regard, l'essentiel est l'idée que l'âme puisse agir à distance, sans contact, c'est elle qui est condamnée.

En dépit de cette sentence, l'exemple du chameau est encore repris par d'autres auteurs, par exemple par Pietro d'Abano, qui le signale de façon évasive dans le Conciliator (1310), sans préciser que cet exemple a fait l'objet d'une condamnation ${ }^{39}$. Plus tard, dans la seconde moitié du XIV siècle, Nicole Oresme adopte une tout autre position. D'un côté, dans le De configuratione, il se réfère aux articles condamnés en 1277 pour réfuter Avicenne (ibn Sīnā, 980-1037), mais sans parler du chameau ${ }^{40}$. D'un autre côté, dans le De causis mirabilium, il attribue à Avicenne un exemple qui se rapproche beaucoup du chameau d'Algazel : Oresme se demande comment Avicenne savait « si ce qu'il affirmait était vrai, à savoir que l'imagination fait tomber une mule ${ }^{41}$. Or on ne trouve pas de mule chez Avicenne! Oresme, qui remplace le chameau par une mule, a sans doute puisé l'exemple chez Algazel. Mais là encore, le rôle du regard est occulté : la chute de l'animal ne concerne pas directement la fascination, elle vient illustrer la thèse d'Avicenne sur le pouvoir de l'âme et de l'imagination.

Voilà nos quatre exemples pour illustrer le pouvoir maléfique du regard. Chaque occurrence met en jeu un élément moteur : un état d'impureté, une pensée chargée de haine ou de colère, la volonté de nuire à quelqu'un. L'âme possèderait alors une puissance destructrice qui lui permettrait d'atteindre un corps différent du sien. Certes, les mécanismes d'action qui sont mis en œuvre sont différents d'un exemple à l'autre ; la fascination apparaît toutefois, dans tous les cas, comme l'expression d'un pouvoir de l'âme au-delà du corps propre. Le phénomène pose problème dans la mesure où il est effectué à distance, par le seul moyen du regard, ce qui va à l'encontre de la définition de l'action naturelle par le contact.

Dans ce cadre, l'exemple de la chute du chameau a une valeur emblématique. Pour Algazel, cette anecdote se rapporte à la fascination. Mais dans les débats du monde latin, elle est surtout utilisée pour illustrer le pouvoir de l'âme. L'exemple de la chute du chameau met ainsi en évidence, dans les débats intellectuels du XIII ${ }^{e}$ et du XIV siècle, de fortes connexions entre le concept de fascination d'une part, et d'autre part l'idée développée par Avicenne d'un pouvoir de l'âme en dehors du corps.

Avicenne et le pouvoir de l'âme

23 La pensée d'Avicenne a exercé une profonde influence sur la scolastique latine à partir du XIII ${ }^{e}$ siècle. La doctrine du pouvoir de l'âme en dehors du corps est exposée dans l'encyclopédie philosophique d'Avicenne, le Kitāb al-shifā (Livre des guérisons [de l'âme]), au sixième et au huitième livre. Ces deux ouvrages ont été traduits en latin, dans les années 1150-1166 pour le De anima, vers 1227 pour le De animalibus ${ }^{42}$. Les thèses avicenniennes constituent l'arrière-plan et le cadre des débats médiévaux sur la fascination.

La psychologie d'Avicenne s'appuie d'abord sur une conception de l'âme étroitement liée au corps ${ }^{43}$. Le corps est l'instrument de l'âme, il contribue à sa perfection. Les affections du corps touchent l'âme intimement, et inversement les affections de l'âme ont un effet direct sur le corps. L'âme peut donc agir sur le corps par la pensée. Le thème est exposé et illustré dans le De anima. Un homme peut marcher sur un tronc posé en travers du chemin, écrit Avicenne, mais si ce tronc était posé au dessus d'une rivière, il n'oserait pas s'avancer au-dessus de l'eau parce que l'image de la chute s'imprime dans son esprit : ses membres obéissent à cette image-là et non à l'image 
contraire, qui l'inciterait à marcher ${ }^{44}$. L'exemple met en évidence le rôle de l'imagination, désignée comme l'un des cinq sens internes de l'âme ${ }^{45}$.

Les actions animales sont placées, elles aussi, sous l'influence qu'exerce l'âme sensitive sur le corps propre. Avicenne s'arrête sur le cas de la poule, lorsqu'elle a vaincu un coq au combat : elle se dresse comme si elle était un coq et parfois il lui pousse un ergot à la patte ${ }^{46}$. Cette anecdote fameuse du De animalibus a été largement utilisée pour soutenir l'idée d'un pouvoir de l'âme sur le corps. Le raisonnement d'Avicenne est le même que dans les exemples humains : la violence des pensées de l'âme impose ses effets au corps propre.

Mais le pouvoir de l'âme va au-delà du corps propre : c'est là l'originalité d'Avicenne, c'est aussi la partie la plus contestée de sa doctrine. Elle est exposée dans un passage célèbre du De anima :

Souvent l'âme agit dans un corps étranger comme dans le corps qui lui est propre, c'est l'œuvre de l'œil qui fascine et qui agit par la pensée. Mais lorsque l'âme est droite et noble, la matière du monde lui obéit et subit son action. Et de cette manière elle guérit les malades, affaiblit les méchants, détruit certaines natures et modifie leurs éléments, de telle sorte que pour elle, ce qui n'est pas feu devient feu et ce qui n'est pas terre devient terre, et que par sa volonté arrivent des pluies et des fertilités comme arrivent l'engloutissement par la terre et les mortalités ${ }^{47}$.

Ce passage distingue deux niveaux dans l'action de l'âme sur le monde. Toute âme peut agir sur les corps étrangers par la fascination; lorsqu'elle est pure, elle peut aussi exercer une influence plus vaste sur le monde extérieur ${ }^{48}$. La matière, conclut Avicenne, est en tout obéissante à l'âme.

Dans l'Occident médiéval, le nom d'Avicenne et la mention du De anima ont été souvent associés à celui d'Algazel, en référence à sa Physica. Algazel a rédigé au $\mathrm{XI}^{\mathrm{e}}$ siècle deux sommes philosophiques en langue arabe pour réfuter les systèmes philosophiques de ses prédécesseurs. Dans la première, le Makācid al-falāsifa (Les intentions des Philosophes), il expose les grands principes philosophiques d'al-Farabī et d'Avicenne; dans la seconde, le Tahāfut al-falāsifa (Les erreurs des philosophes), il s'oppose aux vingt thèses principales de ces deux philosophes.

Dans le monde latin, la philosophie d'Algazel a été souvent confondue avec celle d'Avicenne, bien qu'il soit en contradiction avec elle et qu'il s'emploie à en démonter les principes. Pourquoi ce malentendu? La lecture latine d'Algazel sépare les deux ouvrages complémentaires, Makācid et Tahāfut. Le premier, Makācid, a été traduit en latin à Tolède dans la deuxième moitié du XII ${ }^{e}$ siècle, amputé du prologue et de la conclusion dans lesquels l'auteur exposait ses intentions. Le second, Tahāfut, ainsi que les autres œuvres d'al-Ghazzālī, n'ont pas été traduits. Le Makācid al-falāsifa est donc l'unique ouvrage d'Algazel accessible dans les universités médiévales ${ }^{49}$; il a circulé en latin sous le titre de Summa theoricae philosophiae. L'Occident médiéval n'a connu d'alGhazzālī que son exposé des doctrines d'Avicenne et d'al-Fārābī ; la portée critique de son œuvre est restée méconnue. L'interprétation d'Oresme, qui cite conjointement Avicenne et Algazel sur la fascination, doit être comprise à la lumière de ce malentendu.

La Summa theoricae philosophiae d'Algazel comprend trois parties: une logique, une métaphysique et une physique. Dans la Physica, Algazel rassemble les propositions d'Avicenne sur le pouvoir de l'âme. Le corps propre est présenté comme le domaine d'influence par excellence de l'âme humaine. Le pouvoir de l'âme peut néanmoins se 
porter aussi sur un corps extérieur, comme dans l'exemple du chameau: "parfois, l'impression (impressio) d'une autre âme se transmet à un autre corps, de telle sorte qu'elle détruit l'esprit par une pensée et tue l'homme par la pensée, et cela s'appelle la fascination ${ }^{50} »$. C'est cette conception du pouvoir de l'âme qui a été transmise en Occident par la traduction latine du De anima d'Avicenne ainsi que le résumé qu'en donne Algazel ; c'est elle qui est au centre des réflexions du monde scolastique sur la fascination.

L'influence d'Avicenne en Occident

31 Guillaume d'Auvergne est l'un des premiers penseurs chrétiens à avoir été influencé par Avicenne, en même temps qu'il combattait avec vigueur sa doctrine. Il est aussi considéré comme l'un des premiers lecteurs d'Aristote en Occident. Cette double référence est manifeste dans son interprétation de la fascination. Dans le De Universo, il utilise la notion d'impression comme une clé pour l'explication naturelle de la fascination : le serpent basilic serait «placé par la vision de l'homme dans une telle colère qu'il produit en l'homme une impression mortelle ${ }^{51}$. Le contact des impressions véhiculées par le regard jusqu'au corps de la victime remplace le contact corporel nécessaire à toute action naturelle. Guillaume d'Auvergne tente ainsi de rétablir une causalité par contact dans l'interprétation du phénomène, ce qui préserve la définition aristotélicienne de l'action naturelle.

Dans les années 1240-1270, Roger Bacon est le penseur qui, dans l'Occident médiéval, possède la meilleure connaissance de l'œuvre d'Avicenne. Dans l'opus maius, il explique la fascination par la complexion de l'homme qui regarde, celle-ci étant elle-même rapportée à des facteurs astrologiques. Une mauvaise complexion se propage par l'émission d'impressions, sur le modèle de la contagion. Bacon justifie de cette façon le pouvoir de la femme menstruée ou du basilic: la fascination serait un phénomène naturel de contagion par les yeux. «Et toutes ces choses sont naturelles " ${ }^{52}$, souligne-til en s'appuyant sur le De animalibus et le De anima : les idées d'Avicenne ont donné à Roger Bacon les instruments conceptuels nécessaires à une explication naturelle à la fascination.

Cependant, les idées d'Avicenne ont suscité bien des réticences et des réserves dans le monde latin. Elles étaient visées par deux articles de la condamnation prononcée par l'évêque de Paris Étienne Tempier en 1277 à l'encontre de la faculté des arts de l'Université de Paris : l'article 112 qui, on l'a vu, mentionne l'exemple du chameau, et l'article 210 qui nie que la matière extérieure obéisse à la substance spirituelle ${ }^{53}$. Les censeurs ont repris l'expression d'Avicenne, materia oboediet animae (De Anima, IV, 4). La condamnation portait sur le caractère nécessaire et absolu de la dépendance de la matière à l'âme. Avant et surtout après cette condamnation, la doctrine d'Avicenne fit l'objet d'objections dans le monde occidental. C'est dans ce cadre qu'il faut placer les deux réfutations que nous nous proposons d'analyser ici : celle de Thomas d'Aquin d'abord, dans les années 1269-1272; celle de Nicole Oresme ensuite, dans les années 1351-1362. L'un et l'autre prennent appui sur une réfutation d'Avicenne pour construire une nouvelle interprétation de la fascination.

La pensée d'Avicenne a eu une grande influence sur Thomas d'Aquin, tout en suscitant beaucoup de réserves. Thomas d'Aquin prend la doctrine d'Avicenne comme point de départ de sa démonstration. Il résume les principaux éléments du De anima : le pouvoir de l'imagination en dehors du corps propre, l'importance de la force et de la pureté de l'âme, l'absence de contact corporel et les domaines d'application de cette virtus, en 
médecine et en météorologie ${ }^{54}$. Contre Avicenne, Thomas d'Aquin montre que l'âme ne peut exercer aucune altération directe sur les corps extérieurs : la présence d'un agent corporel est indispensable pour faire le lien entre le moteur de l'action (la substance spirituelle) et son objet (la matière) ${ }^{55}$. Ce principe a sa justification : omne agens agit sibi simile, «tout agent agit sur son semblable " ${ }^{56}$. C'est la définition aristotélicienne de l'action naturelle qui guide le raisonnement de Thomas d'Aquin : «l'âme est incapable, par sa puissance naturelle, de modifier la matière corporelle, sauf par l'intermédiaire de quelques corps ${ }^{57}$.» Thomas d'Aquin se fonde ainsi sur Aristote pour réfuter Avicenne : toute action naturelle sur la matière est une action par contact. L'âme, en tant que substance spirituelle, ne dispose d'aucun pouvoir immédiat sur la matière corporelle.

35 Nicole Oresme condamne lui aussi les doctrines attribuées à Avicenne et Algazel. Il le fait parfois sans argumenter ${ }^{58}$; d'autres fois, son analyse est plus étoffée. Dans le chapitre 38 du De configuratione, la doctrine d'Avicenne est dénoncée en quelques mots incisifs : «Que cela soit fait par l'âme par sa seule pensée est excessivement absurde et irrationnel ; c'est aussi éloigné de la philosophie et dissonant avec notre foi ${ }^{59}$. » La sentence est tranchante, elle articule les deux principaux arguments d'Oresme contre l'action à distance : l'opinion d'Avicenne serait à la fois hostile à la foi chrétienne et contraire à la philosophie. Sur le premier point, Oresme se réfère aux condamnations de 1277 , plus de soixante-dix ans après leur promulgation. Il reprend l'argument en 1370 dans le De causis mirabilium : les thèses d'Avicenne auraient été destinées à prouver que les miracles chrétiens n'étaient que des phénomènes naturels ${ }^{60}$. Cet argument stigmatise en Avicenne un philosophe non chrétien et constitue pour Oresme une première ligne de réfutation.

Le second argument d'Oresme dénonce la doctrine avicennienne comme contraire à la philosophie. Il prend appui d'abord sur la question des altérations intermédiaires. Dans le De configuratione, Oresme présente la thèse d'Avicenne en ces termes: dans le processus de fascination, l'âme commanderait à la matière du monde sans aucune altération intermédiaire ${ }^{61}$. La notion d'altération intermédiaire appartient au processus de la sensation tel qu'Oresme le définit dans le De causis mirabilium ${ }^{62}$. La communication âme-corps, explique-t-il, se déroule en plusieurs étapes : à partir de l'objet, point de départ de la sensation, plusieurs éléments interviennent qui sont d'abord mus, puis deviennent à leur tour mobiles et moteurs. L'influence de l'âme sur le corps passe donc par des éléments médiateurs, les impressions (species) émises par l'objet et les esprits (spiritus) émis en retour par l'âme. Se dispenser des altérations intermédiaires et concevoir une action directe de l'âme sur un corps qui lui est étranger, ce sont là, pour Oresme, des propositions sans fondements.

Le vocabulaire d'Oresme appelle quelques explications. Le terme de species, d'une part, caractérise le mode de perception sensible. Dans son acception première, il désigne l'aspect extérieur d'un objet. Appliquées à la connaissance sensible, les species sont les images de l'objet naturel, émises par lui et qui exercent une impression sur un récepteur. L'optique est le premier domaine d'application de cette théorie: au XIII ${ }^{\mathrm{e}}$ siècle, la notion de species permet d'expliquer la propagation de la lumière par une succession d'impressions corporelles qui se transportent depuis l'émetteur jusqu'au récepteur ${ }^{63}$. Le vocable spiritus, d'autre part, renvoie à la doctrine galénique : l'esprit $(\pi v \varepsilon v \mu \alpha)$ serait un élément corporel de nature subtile remplissant diverses fonctions dans le corps. Dans le monde latin, à partir du XII siècle, la notion fit l'objet de diverses 
réflexions scientifiques, philosophiques et théologiques. Le terme était extrêmement polysémique et couvrait des domaines étendus. Dans un sens biologique, il désignait les éléments corporels qui servent d'intermédiaire entre l'âme et le corps ${ }^{64}$.

Oresme avance encore d'autres arguments pour montrer que la doctrine d'Avicenne est contraire à la philosophie. Dans le De causis mirabilium, il analyse la fascination comme un cas particulier de transmission de pensée ${ }^{65}$. La pensée est ici envisagée sur le modèle de la perception sensorielle; elle est comprise comme l'émission, puis la réception d'une impression de l'âme. Ce phénomène, dit-il, n'est pas plus merveilleux qu'un autre phénomène naturel, d'autant plus que souvent, il s'explique par de simples coïncidences. La seule façon d'envisager une action à distance serait de l'interpréter sur le mode de la transmission de pensée: un homme pourrait, en me transmettant ses species, m'amener à connaître, à vouloir ou à souhaiter la même chose que lui, j'en viendrais à agir de la même façon que lui. Il devient alors concevable de transmettre, avec une pensée, l'effet et le mouvement que cette pensée est censée provoquer. Mais ce n'est là qu'une éventualité : l'homme est libre et il n'agit de cette manière que si cela lui plait. La transmission de pensée est donc conçue comme une forme d'influence indirecte, sans contrainte de l'âme ou du corps qui conservent leur entière liberté de mouvement. Ce qui est écarté par Oresme, c'est qu'une âme puisse agir directement sur un corps à distance en le contraignant à effectuer un certain mouvement. La fascination est ainsi vidée de son sens et ramenée à une forme subtile de persuasion.

Oresme oppose enfin la thèse d'Avicenne à la philosophie aristotélicienne. Sur ce point, il argumente de la même manière que Thomas d'Aquin: les deux raisonnements invoquent la définition de l'action naturelle par le contact, ce qui permet de réfuter l'idée d'un pouvoir direct de l'âme sur un corps étranger. Oresme développe longuement cette réfutation dans le De causis mirabilium ${ }^{66}$, et donne l'exemple du chameau. Dire qu'une thèse est en contradiction avec Aristote constitue pour lui un argument important.

Comment s'accommoder d'Avicenne

Quand Thomas d'Aquin puis Nicole Oresme prennent position contre Avicenne, ils en viennent l'un et l'autre à élaborer une autre manière d'expliquer la fascination. Leurs propositions tentent de nuancer et d'aménager la doctrine d'Avicenne.

41 L'interprétation de Thomas d'Aquin consiste à rétablir un intermédiaire corporel dans la description de la fascination; il s'attache à préciser le trajet de l'altération depuis l'âme jusqu'au corps extérieur. Les relais de l'altération de l'âme sont le cœur, les esprits corporels, puis l'air, dans la limite de la portée du regard. Dans ce cadre, la fascination est décrite comme une suite d'altérations corporelles, elle n'est qu'un cas particulier de l'action naturelle d'un corps sur un autre corps. La fascination n'est plus conçue comme une action à distance ${ }^{67}$. Le raisonnement de Thomas d'Aquin rebondit cependant à propos de la fascination exercée par les vetule : une intervention du démon est possible, ce serait une solution de rechange pour l'explication naturelle de la fascination. Une cause naturelle et une cause démoniaque peuvent s'additionner pour produire des effets supérieurs ${ }^{68}$. Cette interprétation démoniaque n'est pas une concession dans la réflexion de Thomas d'Aquin sur les phénomènes de fascination : les démons occupent une place importante dans sa conception des prodiges.

C'est surtout chez Oresme que la démarche pour s'accommoder d'Avicenne prend toute son ampleur. Le De configuratione revient à la fascination et à l'idée qu'elle mettrait en œuvre le pouvoir de l'imagination, mais Oresme le fait d'une autre façon qu'Avicenne : 
il se réfère à la doctrine des configurations qu'il a développée dans les chapitres précédents et qui détermine son interprétation naturaliste des prodiges ${ }^{69}$. C'est par ce biais qu'il fournit une explication naturelle au pouvoir du basilic et à celui de la femme menstruée. Selon Oresme, l'imagination peut être intensifiée et configurée de manière particulièrement puissante, ce qui donne à l'âme le pouvoir de placer son propre corps dans une certaine disposition. L'altération subie par le corps propre peut ensuite se propager jusqu'à un corps proche : le corps propre la transmet à l'air ou à tout autre médium, et celui-ci la transmet pour finir au corps étranger. La bonne disposition du corps récepteur est une condition importante pour que l'opération réussisse ${ }^{70}$.

L'explication produite par Oresme est surprenante dans la mesure où le lecteur a l'impression qu'elle répète simplement le raisonnement d'Avicenne. De part et d'autre, il est question du pouvoir qu'exerce l'âme, par sa faculté imaginative, sur un corps étranger et distant. Mais l'apport d'Oresme n'est pas négligeable. Il ajoute certaines étapes dans le cheminement du processus d'influence: le mouvement ne passe pas directement de l'âme au corps étranger, il fait intervenir une série de relais intermédiaires, le corps propre et le médium. L'interprétation physique de la fascination que propose Oresme met en jeu une série d'altérations qui maintiennent un contact continu entre l'émetteur et le récepteur. Le facteur central serait alors le médium, qui ramène en quelque sorte l'action à distance au même rang qu'une simple action par contact. Pourtant le texte d'Oresme passe sur cette innovation sans le moindre commentaire et sans même préciser la nature de l'élément médiateur. Plus qu'une doctrine nouvelle, l'explication oresmienne serait une sorte d'amendement à la doctrine d'Avicenne: elle aménage cette doctrine à la lumière des conceptions aristotéliciennes sur le contact.

Les pages suivantes apportent quelques précisions sur cette analyse de la fascination: elle a pour vecteur un rayon visuel, elle passe par les yeux qui sont comme un canal, une voie d'accès reliant les organes des sens internes au monde extérieur. Un contact s'établit en somme lorsque le rayon visuel de l'émetteur est reçu dans l'ouverture oculaire du récepteur : le médium qui transporte l'altération correspond à la tension du regard. Dans les aménagements qu'il apporte à la thèse d'Avicenne, Oresme dessine le profil d'une action à distance qui serait naturelle, par l'intermédiaire de l'imagination, dans une construction qui ne renie pas la définition aristotélicienne de l'action naturelle par le contact.

Nicole Oresme achève son exposé sur la fascination par un retour en arrière un peu déconcertant. Les dernières phrases du chapitre tempèrent l'explication naturaliste de la fascination et remettent en cause le pouvoir de l'imagination: Oresme multiplie les restrictions et les précautions pour qu'une opération de fascination soit considérée comme une action naturelle. Il estime que la fascination est un phénomène rare et marginal, même si ce mode d'action est éventuellement possible ${ }^{71}$. Le recul d'Oresme s'explique sans doute par sa grande prudence et par sa volonté de limiter au maximum la notion de prodige. Mais l'emprise du doute ne remet pas en cause l'explication naturelle de l'action à distance, elle n'en rejette pas les fondements, elle ne fait que limiter au maximum son champ d'application. Les restrictions ultimes d'Oresme sont aussi révélatrices de sa manière de raisonner : le plus important est qu'un phénomène soit possible, sa fréquence ou sa rareté n'ont pas d'incidence profonde sur l'élaboration d'une philosophie naturelle ${ }^{72}$. 
La psychologie de la fascination selon Avicenne a fourni au Moyen Âge les instruments conceptuels d'une interprétation naturelle. Elle a été un élément essentiel du débat sur l'action à distance. La notion d'impressions émises par l'âme était déjà exposée au début du XIII siècle chez Guillaume d'Auvergne. À partir de l'Opus maius (1266-1268), la référence à Avicenne est devenue explicite: Roger Bacon s'appuie sur la doctrine avicennienne pour élaborer son interprétation de la fascination. Mais les thèses d'Avicenne ont été un objet de discorde. Thomas d'Aquin au XIII ${ }^{e}$ siècle, Nicole Oresme au XIV ${ }^{e}$ siècle ont développé à son égard une attitude critique : ils réfutent fermement Avicenne et tentent de dépasser sa doctrine en proposant une autre interprétation de la fascination.

47 L'utilisation latine de la doctrine d'Avicenne va plus loin que la question de la fascination: elle a aussi une incidence dans les débats médiévaux sur le pouvoir des incantations. Certes, la discussion sur l'action à distance s'est concentrée sur le contact visuel et ses modalités ; mais les mêmes raisonnements ont été repris pour interpréter le pouvoir des mots. La réflexion sur la fascination mettait à la disposition des théologiens et des philosophes un cas d'école pour penser le pouvoir de l'incantation comme un pouvoir naturel. Le pouvoir du regard et le pouvoir des mots ont posé le même problème scientifique aux universités du Moyen Âge : le problème de l'action à distance, sans contact. Guillaume d'Auvergne, Roger Bacon, Thomas d'Aquin et Nicole Oresme ont en commun de s'être intéressés aux deux aspects du problème : leurs écrits illustrent avec éclat les liens profonds qui unissaient, dans le monde médiéval, le fascinator qui trouble par son regard et l'incantator qui trouble par ses mots.

\section{NOTES}

1. Voir T. GREGoRY, «La nouvelle idée de nature et de savoir scientifique au XII siècle ", dans J.-E. MURDOCH et E.-D. SYLLA éd., The cultural context of Medieval learning. International Colloquium on Philosophy, Science and Theology in Middle Ages, Boston-Dordrecht, 1975, p. 193-218.

2. ARISTOTE, Physica, III, 202a3 et VII, 242b24, dans ThOMAS D'AQUin, In octo libros Physicorum Aristotelis expositio, P. M. MAGGiolo éd., Rome, 1954, p. 152 et 454.

3. Voir M.-T. D'ALVERNY, «Translators and Translations », dans R. L. BENSON et

G. CONSTABLE éd., Renaissance and Renewal in the twelfth century, Cambridge, Mass., 1982

p. 421-462.

4. Pour une mise en perspective de la question de l'action à distance depuis l'Antiquité jusqu'à l'époque moderne, voir B. P. COPENHAVER, «A Tale of Two Fishes : Magical Objects in Natural History from Antiquity through the Scientific Revolution ", Journal of the History of Ideas 52 (1991), p. 373-398, ainsi que F. J. KovACH, « The Enduring Question of Action at Distance in saint Albert the Great ", dans F. J. KovaCH et R. SHAHAN éd., Albert the Great. Commemorative Essays, Norman, 1980, p. 161-235, en particulier p. 161-171. 
5. Guillaume D'AuVergne, De universo, I, i, 46 et II, i, 32, Opera omnia, Orléans-Paris, 1674, vol. I, p. 657-658 et 833-834.

6. Roger BACon, Opus maius, IV, J. H. BRIDGES éd., Oxford, 1897, vol. I, p. 142-143 et 398.

7. Thomas D'AQUin, Contra gentiles, III, 103 et Summa theologica, I, 117, dans Opera omnia, Rome, 1926.

8. Nicole ORESMe, De configuratione qualitatum, II, 37-38, M. CLAGETT éd., Madison, 1968, p. 376-386.

9. ID., De causis mirabilium, IV, nota 14 et 19, B. HANSEN éd., Toronto, 985, p. 314 et 354.

10. Voir B. Delaurenti, Virtus verborum. Débats doctrinaux sur le pouvoir des incantations au $\mathrm{XIII}^{e}$ et au XIVe siècle, Paris, thèse de Doctorat à l'EHESS, 2004, ainsi que C. FANGER, « Things done wisely by a wise enchanter : negotiating the power of words in the Thirteenth Century ", Esoterica : the Journal of Esoteric Studies, I (1999), p. 97-132.

11. Pietro D'ABAno, Conciliator, diff. 64 et 135, Venise, $1565,94 \mathrm{vaH}$ et 192vaE. Voir

E. PAschetTo, Pietro d'Abano, medico e filosofo, Florence, 1984, p. 136-138.

12. Sur la fascination du point de vue médical, voir F. SALMÓN et M. CABRÉ, « Fascinating women : the evil eye in medical scholasticism ", dans R. FRENCH et al., Medicine from the Black Death to the French Disease, Aldershot, 1998, p. 53-84. Pour une approche anthropologique de la fascination, voir C. MALONEY dir., The evil eye, New-York, 1976.

13. Biblia latina cum glossa ordinaria, Brepols, 1992, repr. de l'édition de Strasbourg 1480, p. 359.

14. Pline, Historia naturalis, VIII, 33, E. DE SAINT-DENIS éd., Paris, 1955, p. 50-51.

15. ID., XXIX, 66, p. 41-42.

16. LUCAIN, La guerre civile (La Pharsale), IX, 724-726 et 828, A. BOURGERY éd., Paris, 1976,

p. 163-164 et 168 .

17. Solin, Collectanea rerum memorabilium, 27, 50, T. MOMMSEN éd., Berlin, 1895, p. 126-127.

18. AleXANDER NeCKham, De naturis rerum, 120, T. Wright éd., Londres, 1863, p. 198.

19. ThOMAS DE CANTIMPRÉ, De natura rerum, VIII, 4, vol. I, H. BoESE éd., Berlin, 1973, p. 278-279.

20. VINCENT DE BEAUVAIS, Speculum naturale, XX, 22, 23 et 41, dans Bibliotheca mundi Vincentii, Douai, 1624, repr. Graz 1965, vol. I, p. 1473, 1474 et 1481.

21. Guillaume D'Auvergne, De universo, II, iii, 16, op. cit., p. $1046 \mathrm{aG}$; ibid., I, i, 46, p. $657 \mathrm{aD}-658 \mathrm{~b}$.

22. ROGER BACON, Opus maius, IV, op. cit., p. 142-143 : « per proprium destrueretur venenum ".

23. L.-L. GunDERSON, Alexander's Letter to Aristotle about India, Meisenheim am Glan, 1980, p. 53-54.

24. Epistola Alexandri ad Aristotelem, dans W. W. BOER, Beiträge zur klassischen Philologie 50, 1973, p. 18 : « Binorum ternorumque capitum cristati serpentes indici columnarum grossitudine aliquantulum proceriores (...) ; cum trisulcis lingui fauces exserebant scintillantibus veneno oculis ; quorum halitus quoque erat pestifer. "

25. Guillaume D'Auvergne, De universo, II, i, 32, op. cit., p. 833.

26. OVIDE, Les amours, I, 8, 1. 15-18, H. BORNECQUE éd., Paris, 1989.

27. Roger BACon, Epistola..., J. S. BREWER éd., Londres, 1859, vol. I, p. 529.

28. Nicole ORESME, De configuratione, II, 28, op. cit., p. 344.

29. ARISTOTE, De somniis, 459b 27-460a 26, Paris, 1965, p. 80-81.

30. RogER BACON, Opus maius IV, op. cit., p. 398. 
31. Sur la nocivité du sang menstruel, voir D. JACQUART et C. THOMASSET, Sexualité et savoir médical au Moyen Âge, Paris, 1985, p. 103-106 et F. SALMÓN et M. CABRÉ, loc. cit.

32. ThomAS D'AQuin, Contra gentiles, III, chap. 103, n. 5, op. cit. ; Summa theologica, I, q. 117 a. 3 ad 2., op. cit.

33. Nicole ORESME, De configuratione, II, 38, op. cit., p. 384.

34. Virgile, Bucoliques, III, v. 100-104, Paris, E. DE SAINT-Denis éd., Paris, 1992 : « Nescio quis teneros oculus mihi fascinat agnos ».

35. ROGER BACON, Opus maius, IV, op. cit., p. 398 ; NICOLE ORESME, De configuratione, II, 38, op. cit., p. 382.

36. Algazel, Physica, V, 9, J. T. Muckle éd., Toronto, 1933, p. 194 : « Oculus mittit hominem in fossam, et camelum in caldarium, et dicitur quod homines fascinari. »

37. Guillaume D'AuVERgne, De universo, II, iii, 16, op. cit., p. $1046 \mathrm{bE}$.

38. Article 112 dans D. PICHÉ éd., La condamnation parisienne de 1277, Paris, 1999, p. 112.

39. Pietro D'Abano, Conciliator, diff. 135, 192vaE.

40. Nicole ORESME, De configuratione, II, 38, op. cit., p. 382.

41. ID., De causis mirabilium, IV, nota 14, op. cit., p. 314 : « Quid autem scivit Avicenna utrum illud quod ibi ponit sit verum, scilicet quod ymaginatio fecit cadere mulum etc?».

42. Voir D. N. HASSE, Avicenna's De anima in the Latin West, Londres, 2000 ; A.-M. GoICHON, La philosophie d'Avicenne et son influence en Europe médiévale, Paris, 1984 ; G.-C. ANAWATI dans AvicEnNE, La métaphysique du Shïfā', Paris, 1978. Sur les traductions latines d'Avicenne, voir M.-T. D'AlVERnY, Avicenne en Occident, Paris, 1993.

43. P. RolAND-Gosselin, «Les relations du corps et de l'âme d'après Avicenne ", dans Mélanges Mandonnet. Études d'histoire littéraire et doctrinale du Moyen Âge, Paris, 1930, vol. 2, et S. VAN RIET, « Une conception spiritualiste de l'homme », Avicenna latinus : Liber de Anima sive sextus De Naturalibus, Louvain, 1972, t. II, p. 36*-46*.

44. AVicenne, De anima, IV, 4, S. VAN RiET éd., p. 64.

45. Voir E.-R. HARVEY, The inward wits. Psychological theory in the Middle Âges and Renaissance, Londres 1975 ; H.-A. WolfSON, « The internal senses in Latin, Arabic and Hebrew psychological texts ", Harvard Theological Review, 28, 1935, p. 69-73. Sur la question du pouvoir de l'imagination, voir L. THORNDIKE, « Imagination and Magic. The Force of Imagination on the Human Body and of Magic on the Human Mind », Mélanges Eugène Tisserant, VII, Cité du Vatican, 1964, p. 353-358, ainsi que P. ZAMBELLI, "L'immaginazione e il suo potere. Desiderio e fantasia psicosomatica o transitiva », dans EAD., L'ambigua natura della magia : filosofi, streghe, riti nel Rinascimento, Milan, 1991, p. 53-75.

46. AVICENNE, De animalibus VIII, 7, dans Opera philosophica, Venise, 1508.

47. ID., De anima, IV, 4, op. cit., p. 65-66: «Multotiens autem anima operatur in corpore alieno sicut in proprio, quemadmodum est opus oculi fascinantis et aestimationis operantis ; immo cum anima fuerit constans, nobilis, similis principiis, oboediet ei materia quae est in mundo et patietur ex ea, (...) ut sanet infirmos et debilitet pravos et contingat privari naturas et permutari sibi elementa, ita ut quod non est ignis fiat ei ignis, et quod non est terra fiat ei terra, et pro voluntate eius contingant pluviae et fertilitas sicut contingit absorbitio a terra et mortalitas. "

48. D'après D. N. Hasse, op. cit., p. 162-163, l'action d'une âme sur un corps extérieur s'applique au sorcier pratiquant le mauvais œil, tandis que l'action d'une âme pure sur la matière du monde s'applique au vrai prophète. 
49. Il existait néanmoins d'autres voies pour connaître la pensée d'Algazel au Moyen Âge, mais ces voies ont été peu diffusées. Voir D. SALMAN, « Algazel et les latins », Archives d'Histoire Doctrinale et Littéraire du Moyen Âge, 10, 1935-1936, p. 103-127.

50. AlgAZel, Physica, V, 9, op. cit., p. 194 : « Aliquando autem impressio alicuius anime pertransit ad aliud corpus, sic ut destruat spiritum estimacione, et inficiat hominem estimacione, et hoc dicitur fascinatio. $»$

51. Guillaume D'Auvergne, De universo., I, i, 46, op. cit., p. 657aD-658b : « Quorum primum exemplum pono tibi basilisci serpentis (...). Commovetur enim aspecto humano in tantam iram, et talem, quod impressionem mortis facit in homine. "

52. ROGER BACON, Opus maius, IV, op. cit., p. 398 : « Et haec naturalia sunt.»

53. Article 210, D. PichÉ éd., p. 144.

54. Thomas D'Aquin, Contra gentiles, III, 103 n. 1 et 2 et Summa theologiae, I, q. 117 a. 3 ad 2, op. cit.

55. ID., Contra gentiles, III, 103, n. 6, co. et Summa theologiae, I, q. 117, art. 3.

56. ID., Summa theologiae, I, q. 110, art. 2, resp.

57. ID., q. 117 a. 3 co. : « Multo igitur minus anima sua virtute naturali potest immutare materiam corporalem, nisi mediantibus aliquibus corporibus. »

58. NICOLE ORESME, De configuratione, II, 28 et 35 , op. cit.

59. ID., II, 38, p. 382 : « Sed hoc fieri ab anima per solam cogitationem est nimis absurdum et irrationabile (...) ; est etiam remotum a philosophia et fidei nostrum dissonum. »

60. Nicole ORESME, De causis mirabilium, IV, nota 19, op. cit., p. 354.

61. ID., De configuratione qualitatum, II, 38, op. cit., p. 382.

62. ID., De causis mirabilium, IV, nota 19, op. cit., p. 346.

63. Voir P. MichAUD-QuANTIN, « Les champs sémantiques de species. Tradition latine et traductions du grec », Études sur le vocabulaire philosophique au Moyen Âge, Rome, 1970, p. 113-150. Sur la notion de species en optique, voir D.-C. LINDBERG, Theories of vision from Al Kindy to Kepler, Chicago, 1976, p. 104-121 et K.-H. TACHAU, Vision and certitude in the Age of Ockham. Optics, Epistemology and the Foundations of Semantisc (1250-1345), Leyde, 1988.

64. Voir M.-D. CHENU, «Spiritus : vocabulaire de l'âme au XII siècle ", Revue des Sciences Philosophiques et Théologiques, 41, 1957, p. 209-32 et J. BoNo, « Medical Spirits and the medieval Language of Life », Traditio, 40, 1984, p. 91-130.

65. NICOLE ORESME, De causis mirabilium, IV, nota 14, op. cit., p. 310-314.

66. Ibid., p. 314 et nota 19, p. 354 .

67. THOMAS D'AQUIN, Contra gentiles, III, 103 n. 5 et Summa theologica, I, q. 117 a. 3 ad 2, op. cit.

68. ID., Contra gentiles, III, 103, n. 8, et Summa theologiae, I, q. 117 a. 3 ad 2.

69. Sur la doctrine des configurations, voir M. CLAGETT, Nicole Oresme and the Medieval Geometry of Qualities and Motions, Madison, 1968, p. 14-50 ; A. MAIER, « La doctrine d'Oresme sur les configurationes intensionum ", dans Ausgehendes Mittelalter I: Gesammelte Aufsätze zur Geistesgeschichte des 14. Jahrhunderts, Rome, 1964, p. 335-352.

70. NICOLE ORESME, De configuratione, II, 38, op. cit., p. 382-384.

71. Ibid., p. 384-86.

72. Sur le probabilisme d'Oresme, voir S. CAROTI, «Éléments pour une reconstruction de la philosophie de la nature dans les Quodlibeta de Nicole Oresme », dans J. QUILLET éd., Autour de Nicole Oresme, Paris, 1990, p. 85-118 ; H. HugONNARD-ROCHE, « Modalités et 
argumentation chez Nicole Oresme ", dans P. SouffRin, A. SEGONDS dir., Nicolas Oresme, tradition et innovation chez un intellectuel du XIVee siècle, Paris, 1988, p. 145-164.

\section{RÉSUMÉS}

Le terme fascinatio désignait à l'époque scolastique le pouvoir qu'un homme ou un animal pouvait exercer, à distance, par l'intermédiaire de son regard. Ce phénomène prodigieux a suscité les interrogations des auteurs médiévaux : dans quelle mesure la fascination pouvait-elle avoir une cause naturelle? De 1230 à 1370, Guillaume d'Auvergne, Roger Bacon, Thomas d'Aquin puis Nicole Oresme ont intégré cette question dans leur réflexion philosophique sur les pouvoirs de la nature. Il s'agissait pour eux d'adapter les principes de la physique aristotélicienne, qui veut que toute action naturelle s'exerce par contact, à la question controversée de l'action à distance, sans contact. Leurs réflexions montrent comment la définition de l'action naturelle s'est construite et précisée au fil de ces débats : l'idée qu'une action à distance pouvait être une action naturelle a été soutenue d'abord en invoquant le rôle des impressions de l'âme, puis par un aménagement de plus en plus substantiel de la doctrine contestée d'Avicenne sur le pouvoir de l'âme en dehors du corps.

Fascination and action at a distance: medieval questions (1230-1370). In medieval Europe, the latin term fascinatio indicated the power which a man or an animal could possess, at a distance, by its only glance. This extraordinary phenomenon induced some interrogations among medieval authors : up to what point could fascination have a natural cause ? From 1230 to 1370, William of Auvergne, Roger Bacon, Thomas Aquinas and then Nicole Oresme integrated this question in their philosophical reflexion about the powers of nature. The problem was to adapt the principles of the aristotelian physics, which states that any natural action is exerted by contact, with the possibility of an action at a distance, without any contact. Their reflexions show how the definition of the natural action was built and specified during these debates: the idea that an action at a distance could be a natural action was elaborated first by calling upon the role of the impressions of the soul, then by an increasingly substantial specification of the doctrine of Avicenne about the power of the soul outside the body.

\section{INDEX}

Keywords : fascination, Avicenne, Aristotle, naturalism, action at distance

Mots-clés : Aristote, naturalisme, action à distance

\section{AUTEUR}

BÉATRICE DELAURENTI

GAS, EHESS, 54, boulevard Raspail, 75006 Paris 